\title{
MODELLING OF SOLAR CORONAL LOOPS
}

\author{
C. JORDAN \\ Department of Physics (Theoretical Physics) \\ University of Oxford \\ 1, Kcble Road, Oxford OX1 3NP, U.K.
}

\section{Introduction}

Modelling of coronal active regions in terms of loop structures began around 1974 (see Jordan 1975) and was stimulated by images of the corona in X-rays and the uv, obtained from rocket flights. Vaiana was a pioneer in this field (see Vaiana et al. 1973). The Skylab missions provided a fuller range of imaging and spectroscopic observations, and much of the available information is still based on these data (see Orrall 1981). Since 1975 a very large number of papers have been published on the various aspects of loop structure, heating and stability (see review by Mewe 1991). Here I can mention only a few points concerning the relation between observable parameters and the energy balance and heating requirements of active region loops.

\section{Observations}

The range of conditions in active region loops, e.g. maximum temperatures $\left(T_{m} \sim 5 \times 10^{5}\right.$ $\mathrm{K}$ to $\left.3 \times 10^{6} \mathrm{~K}\right)$, electron pressures $\left(P_{\epsilon} \sim 10^{15}\right.$ to $\left.10^{16} \mathrm{~cm}^{-3} \mathrm{~K}\right)$ and lengths $\left(L \sim 10^{9}\right.$ to $2 \times 10^{10} \mathrm{~cm}$ ) are well known from Skylab observations (see reviews in Orrall 1981). Although each loop appears isothermal at some $T_{m}$ there is strong evidence of a temperature gradient across the legs of a given loop, with cooler material lying, on average, within the hotter (Foukal 1975). The higher electron pressures measured from line ratios rather than from emission measures divided by path lengths suggests a structure inhomogeneous in both density and temperature (Dere 1982a). Recent high spatial resolution X-ray observations also support a thread-like structure (Golub 1991). The evidence for lower pressures in loop cores is weaker and further observations are required. This aspect is important in modelling loop stability (Einaudi and Van Hoven 1983). Along a loop hydrostatic equilibrium is usually followed (Cabriel and Jordan 1975, Dere 1982a) although clear examples of flows occur. One must beware that emission from a given line can occur at temperatures above the "optimum" for formation in a uniform atmosphere, when deducing evidence of departures from hydrostatic equilibrium, simply from the presence of a line up to a particular height. It is essential to measure simultaneously the "coronal" and transition region properties of a given loop, and ideally the underlying photospheric magnetic fields. Although the global properties of active regions are being examined in this way from SMM and other data, few such studies of individual loops exist (e.g. Pallavicini et al. 1981) and these lack direct measurements of the density. 


\section{Structure and energy balance}

Many models have been made in terms of a fixed pressure, $P_{o}$, and a heating function of the form $E_{H}=\epsilon_{o} T_{\varepsilon}^{\gamma}$. Solutions to the energy balance equation can be found for the regions above and below $T_{*}$, the temperature at which the conductive flux has a maximum value, so that $E_{H}=\alpha P_{o}^{2} / T_{*}^{-5 / 2}$, the radiative loss rate at $T_{*}$. The base conductive flux, $F_{c}\left(T_{o}\right)$, and $T_{m}$, where $F_{c}\left(T_{m}\right)=0$, must also be specified. Unless $F_{c}\left(T_{o}\right)=0, T_{*}$ depends on $F_{c}\left(T_{o}\right), P_{o}^{2}$, and $T_{m}$, as well as $\gamma$. The loop length involves integrating the general expression for $F_{c}\left(T_{\varepsilon}\right)$. Thus 4 of the 5 parameters must be known, not only $T_{m}, P_{o}$ and $L$. A single power fit to the radiative losses should be used only down to $T_{o} \sim 2 \times 10^{5} \mathrm{~K}$, where the observed emission measures, $\left(\operatorname{Em}\left(T_{o}\right)=\left(P_{o}^{2} \kappa T_{o}^{3 / 2}\right) /\left(2^{1 / 2} F_{c}\left(T_{o}\right)\right)\right.$, show that $F_{c}\left(T_{o}\right)$ is large. Many combinations of fixed and variable parameters, with $2>\gamma>-2$, have been explored in the literature (see Withbroe 1981). In practice the observed emission measure distributions show that $d \log E m / d \log T_{e}$ is close to $3 / 2$ (Dere $1982 \mathrm{~b}$ ) which could imply constant conductive flux from the region where heating occurs. In this case $\gamma=-5 / 2$. Since the method above uses the same $\gamma$ for all $T_{e}$ it cannot be used for $\gamma=-5 / 2$ all the way to $T_{m}$. If $F_{c}\left(T_{m}\right)=0$, a value of $\gamma \neq-5 / 2$ is required in the region of main heating. Because the conductive flux at $T_{o}$ is large, and the radiative losses between $T_{o}$ and $T_{m}$ are small, the case of $F_{c}\left(T_{e}\right)=$ const. gives almost the same emission measure distribution as the case of no heating in this region. We can conclude that the heating occurs only near the peak temperature. The main use of the above theoretical approach is to obtain global scaling laws between $P_{o}, T_{m}$ and $L$. As Hearn and Kuin (1981) have shown, virtually the same results are obtained with a minimum energy loss solution. The ratio $F_{c}\left(T_{*}\right) / F_{r}\left(T_{m}\right)$ is then fixed and solutions with smaller ratios are not stable. The total energy input required is insensitive to how the energy is lost.

The main outstanding problem is to find in detail a process to provide the total heating required and predict its dependence on the plasma parameters and the magnetic field. To exclude, or otherwise, wave processes, the variation of non-thermal line widths with temperature must be simultaneously measured.

\section{References}

Dere, K.P.: 1982a., Sol. Phys., 75, 189.

Dere, K.P.: 1982b, Sol. Phys., 77, 77.

Einaudi, G. \&. Van Hoven, G.: 1983, Sol. Phys., 88, 163.

Foukal, P.: 1975, Sol. Phys., 43, 327.

Gabriel, A.H. \& Jordan, C.: 1975, Mon. Not. R. astr. Soc., 173, 397.

Golub, L.: 1991, in 'Mechanisms of Chromospheric and Coronal Heating', eds. P. Ulmschneider, E.R. Priest \&. R. Rosner, (Springer-Verlag), p. 115.

Hearn, A.G. \& Kuin. N.P.M.: 1981, Astron. Astrophys,, 98, 248.

Jordan, C.: 1975, in 'Solar Gamma, $X$-, and EUV Radiation', ed. S.R. Kane, (Reidel, Dordrecht), p. 109.

Mewe, R.: 1991, Adv. Space Res., Vol. 11, No. 1, p. (1)127.

Orrall, F.Q.: 1981, ed. 'Solar Active Regions', (Colorado Ass. Univ. Press, Boulder CO). Pallavicini, R., Peres, G., Serio, S., Vaiana, G.S., Golub, L. \&. Rosner, R.: 1981, Astrophys. J., 247, 692.

Vaiana, G.S., Kreiger, A.S. \& Timothy, A.F.: 1973, Sol. Phys., 32, 81.

Withbroe, G.: 1981, in 'Solar Active Regions', ed. F.Q. Orrall, (Colorado Ass. Univ. Press, Boulder CO), p. 109. 